\title{
On the finiteness of a new topological model in $D=3$
}

\author{
O.M. Del Cima ${ }^{a *}$, J.M. Grimstrup ${ }^{a, b \dagger}$ and M. Schweda ${ }^{a \ddagger}$ \\ ${ }^{a}$ Institut für Theoretische Physik (ITP), \\ Technische Universität Wien (TU-Wien), \\ Wiedner Hauptstraße 8-10 - A-1040 - Vienna - Austria. \\ ${ }^{b}$ The Niels Bohr Institute (NBI), \\ Blegdamsvej 17 - DK-2100 - Copenhagen Ø - Denmark.
}

(October 4, 2018)

\begin{abstract}
A new topological model is proposed in three dimensions as an extension of the BF-model. It is a three-dimensional counterpart of the two-dimensional model introduced by Chamseddine and Wyler ten years ago. The BFK-model, as we shall call it, shows to be quantum scale invariant at all orders in perturbation theory. The proof of its full finiteness is given in the framework of algebraic renormalization.
\end{abstract}

PACS numbers: 11.10.Gh 11.10.Kk 11.15.-q 11.15.Bt

TUW-99-12

\section{INTRODUCTION.}

The BF-models have been widely investigated since the middle of the eighties [1 12], specially the two-dimensional BF-model in connection with topological quantum gravity 1 迎. The properties of ultraviolet and infrared perturbative finiteness of the two-dimensional BF-model have been rigorously proved in the framework of algebraic renormalization [5], there the authors make use of an additional symmetry peculiar to topological models in the Landau gauge, called vector-supersymmetry [6,13 [15]. As a natural extension of the two-dimensional case, it has been proposed in [3] an extended BF-model, as a gauge theory for topological quantum gravity, which accommodates a topological matter coupling. Later, the authors of [7] have been shown that the model proposed in [3] is still ultraviolet and infrared finite at all orders in perturbation theory by using the same approach as those of [5] have done to the pure two-dimensional BF-model.

At the level of perturbation theory, a general classification of all possible anomalies and invariant counterterms of BF-models in any space-time dimension has been done in [8]. The feature of the independence on the gauge coupling of those models was pointed out by the authors of [9]. Moreover, it has been shown that $D$-dimensional BF-Yang-Mills-models, a BF formulation of the Yang-Mills theory, are cohomologically equivalent to Yang-Mills [10]. Three-dimensional BF-Yang-Mills theory, both Gaussian and extended, have been algebraically quantized by the authors of 11], where the proof of their exact quantum scale invariance at all orders in perturbation theory have been given in 12. Note: after the completion of this work we became aware of ref. [16], where a dimensional reduction $\grave{a} l a$ Scherk of the Gaussian four-dimensional Abelian version of the BF-Yang-Mills-model is performed to three dimensions leading to a model similar to ours, there its spectrum is analyzed in details as well.

Our purpose in this letter is to present a rigorous proof on the finiteness of an extended version of the BF-model in three dimensions introduced here. Such a model, we shall call BFK-model, is a three-dimensional counterpart of that model in two dimensions proposed by Chamseddine and Wyler 3 . The proof is performed by using the method of algebraic renormalization [6], which is independent of any particular regularization scheme. The letter is organized as follows. The BFK-model and its symmetries are introduced in Section II. In Section III, the proof on the full finiteness at all orders in perturbation theory is sketched and at the end the conclusions are drawn. Here in this letter we summarize the main results and all details shall be reported elsewhere in a more complete paper [17]. It is now under investigation the classification of all possible counterterms and anomalies of the $D$-dimensional BFK-model [18] by following the same approach of [8].

\footnotetext{
${ }^{*}$ Supported by the Fonds zur Förderung der Wissenschaftlichen Forschung (FWF) under the contract number P11654-PHY. E-mail:delcima@tph73.tuwien.ac.at.

${ }^{\dagger}$ E-mail:jesper@tph41.tuwien.ac.at.

${ }^{\ddagger}$ E-mail:mschweda@tph.tuwien.ac.at.
} 


\section{THE BFK-MODEL IN $D=3$ AND ITS SYMMETRIES}

\section{A. The classical action}

The classical action of the BFK-model in $D=3$ is given by

$$
\Sigma_{\mathrm{inv}}^{\mathrm{BFK}}=\frac{1}{2} \operatorname{Tr} \int d^{3} x \varepsilon^{\mu \nu \rho}\left\{B_{\mu} F_{\nu \rho}+K_{\mu \nu} D_{\rho} \phi\right\},
$$

where $B_{\mu}$ is a vector field, $K_{\mu \nu}$ is a rank-2 antisymmetric tensor and $\phi$ is a scalar. The second piece of the action (11) can be seen as a topological matter coupling, where the matter fields $K_{\mu \nu}$ and $\phi$ lie in the adjoint representation of the gauge group. In two dimensions similar topological matter term was proposed by Chamseddine and Wyler [3]. Any field, $\varphi$, is to be assumed as Lie algebra valued, in such a way that

$$
\varphi \equiv \varphi^{a} \tau_{a}
$$

where the matrices $\tau$ are the generators of the gauge group 7 and obey

$$
\left[\tau_{a}, \tau_{b}\right]=f_{a b c} \tau_{c} \text { and } \operatorname{Tr}\left(\tau_{a} \tau_{b}\right)=\frac{1}{2} \delta_{a b}
$$

The field strength, $F_{\mu \nu}$, is defined as

$$
F_{\mu \nu}=\partial_{\mu} A_{\nu}-\partial_{\nu} A_{\mu}+\left[A_{\mu}, A_{\nu}\right]
$$

and the covariant derivative reads

$$
D_{\mu} \phi=\partial_{\mu} \phi+\left[A_{\mu}, \phi\right]
$$

\section{B. Gauge symmetries}

The action (11) possesses two symmetries:

1. The standard gauge symmetry

$$
\begin{aligned}
& \delta_{\alpha} A_{\mu}=-D_{\mu} \alpha \equiv-\left(\partial_{\mu} \alpha+\left[A_{\mu}, \alpha\right]\right), \quad \delta_{\alpha} B_{\mu}=\left[\alpha, B_{\mu}\right] \\
& \delta_{\alpha} K_{\mu \nu}=\left[\alpha, K_{\mu \nu}\right] \text { and } \delta_{\alpha} \phi=[\alpha, \phi] .
\end{aligned}
$$

2. The topological symmetry

$$
\begin{aligned}
& \delta_{\beta} A_{\mu}=0, \quad \delta_{\beta} B_{\mu}=-\left(D_{\mu} \beta+\left[\beta_{\mu}, \phi\right]\right) \\
& \delta_{\beta} K_{\mu \nu}=-\left(D_{\mu} \beta_{\nu}-D_{\nu} \beta_{\mu}\right) \text { and } \delta_{\beta} \phi=0
\end{aligned}
$$

\section{BRS symmetry}

The corresponding BRS transformations of the fields, $A_{\mu}, B_{\mu}, K_{\mu \nu}$ and $\phi$, stemming from the symmetries (6) and (7), are given by

\footnotetext{
${ }^{1}$ The gauge group is considered as a general compact one.

${ }^{2}$ The commutators are assumed to be graded, namely, $\left[\varphi_{1}^{g_{1}}, \varphi_{2}^{g_{2}}\right] \equiv \varphi_{1}^{g_{1}} \varphi_{2}^{g_{2}}-(-1)^{g_{1} \cdot g_{2}} \varphi_{2}^{g_{2}} \varphi_{1}^{g_{1}}$, where the upper indices, $g_{1}$ and $g_{2}$, are the Faddeev-Popov charges $(\Phi \Pi)$ carried by $\varphi_{1}^{g_{1}}$ and $\varphi_{2}^{g_{2}}$, respectively.
} 


$$
\begin{aligned}
& s A_{\mu}=-D_{\mu} c \equiv-\left(\partial_{\mu} c+\left[A_{\mu}, c\right]\right), \quad s B_{\mu}=-D_{\mu} B^{1}+\left[\phi, B_{\mu}^{1}\right]+\left[c, B_{\mu}\right] \\
& s K_{\mu \nu}=-\left(D_{\mu} B_{\nu}^{1}-D_{\nu} B_{\mu}^{1}\right)+\left[c, K_{\mu \nu}\right], \quad s \phi=[c, \phi] \quad \text { and } \quad s c=c^{2}
\end{aligned}
$$

where $c$ and $B^{1}$ are scalar ghosts, and $B_{\mu}^{1}$ is a vector ghost, all of them are anticommuting fields with Faddeev-Popov charge (ghost number) one. Bearing in mind that we have a residual degree of freedom from $s K_{\mu \nu}$ caused by a zero mode $\left(B_{\mu}^{1}=D_{\mu} \varphi^{2}\right)$, i.e., it is a reducible symmetry, has to be fixed, therefore, yielding a ghost $B^{2}$ for the ghost $B_{\mu}^{1}$. Now, fixing the zero mode by introducing the ghost for ghost, $B^{2}$, the remaining BRS transformations read

$$
s B^{1}=\left[\phi, B^{2}\right]+\left[c, B^{1}\right], \quad s B_{\mu}^{1}=D_{\mu} B^{2}+\left[c, B_{\mu}^{1}\right] \text { and } s B^{2}=\left[c, B^{2}\right] .
$$

It should be noticed that the BRS operator $s$ is nilpotent up to the field equations for $B_{\mu}$ and $K_{\mu \nu}$, since

$$
s^{2} B_{\mu}=\frac{1}{2}\left[B^{2}, \varepsilon_{\mu \nu \rho} \frac{\delta \Sigma_{\mathrm{inv}}^{\mathrm{BFK}}}{\delta K_{\nu \rho}}\right] \text { and } s^{2} K_{\mu \nu}=\left[B^{2}, \varepsilon_{\mu \nu \rho} \frac{\delta \Sigma_{\mathrm{inv}}^{\mathrm{BFK}}}{\delta B_{\rho}}\right]
$$

called on-shell nilpotency.

\section{Gauge-fixing}

The gauge-fixing we are considering here is of the Landau-type. Since we are dealing with a more complex model than the Yang-Mills one, face its symmetries, some subtleties arise as their consequence. In order to implement the gauge-fixing we couple the Lagrange multiplier fields $b, \pi^{0}, \pi^{0 \mu}$ and $\pi^{-1}$ to

$$
\operatorname{Tr} b \partial^{\mu} A_{\mu}, \quad \operatorname{Tr} \pi^{0} \partial^{\mu} B_{\mu}, \quad \operatorname{Tr} \pi^{0 \nu}\left(\partial^{\mu} K_{\mu \nu}+\partial_{\nu} \rho^{0}\right) \text { and } \operatorname{Tr} \pi^{-1}\left(\partial^{\mu} B_{\mu}^{1}+\lambda^{1}\right),
$$

where the multiplier fields belong to the following BRS-doublets:

$$
\begin{aligned}
s \bar{c} & =b, \quad s b=0 ; \\
s \bar{c}^{-1} & =\pi^{0}, \quad s \pi^{0}=0 ; \\
s \bar{c}^{-1 \mu} & =\pi^{0 \mu}, \quad s \pi^{0 \mu}=0 ; \\
s \bar{c}^{-2} & =\pi^{-1}, \quad s \pi^{-1}=0 .
\end{aligned}
$$

We stress here that for the fields, $K_{\mu \nu}$ and $B_{\mu}^{1}$, inhomogeneous gauge conditions (11) have been chosen. In which concerns the field $K_{\mu \nu}$, a gauge condition of the type $\pi^{0 \nu} \partial^{\mu} K_{\mu \nu}$ would not fix completely the gauge, since a residual degree of freedom is present by $\pi^{0 \nu} \rightarrow \pi^{0 \nu}+\partial^{\nu} \tilde{\rho}^{0}$, therefore, due to this fact an inhomogeneous gauge-fixing condition has to be adopted. Since it is an Abelian transformation does not demand further ghost fields. Besides the condition on $K_{\mu \nu}$ has introduced a new field $\rho^{0}$, it yields also the consideration of another inhomogeneous gauge condition associated to $B_{\mu}^{1}$ by putting into the game the field $\lambda^{1}$. In fact, the introduction of $\lambda^{1}$ is due to cohomological arguments, so in order to protect the independence of BRS-cohomology in those fields introduced by hand, we force them to belong to a BRS-doublet

$$
s \rho^{0}=\lambda^{1}, s \lambda^{1}=0 .
$$

Bearing in mind that a neutral Faddeev-Popov charge action is desired, the fields $\rho^{0}$ and $\lambda^{1}$ have already had their charges fixed 0 and 1 , respectively.

Now, to introduce a BRS-trivial gauge-fixing compatible with the gauge conditions (11), we add to the action (1) the following four pieces:

$$
\begin{aligned}
& \Sigma_{\mathrm{gf}}^{1}=s \operatorname{Tr} \int d^{3} x \bar{c} \partial^{\mu} A_{\mu}, \quad \Sigma_{\mathrm{gf}}^{2}=s \operatorname{Tr} \int d^{3} x \bar{c}^{-1} \partial^{\mu} B_{\mu}, \\
& \Sigma_{\mathrm{gf}}^{3}=s \operatorname{Tr} \int d^{3} x \bar{c}^{-1 \nu}\left(\partial^{\mu} K_{\mu \nu}+\partial_{\nu} \rho^{0}\right) \quad \text { and } \quad \Sigma_{\mathrm{gf}}^{4}=s \operatorname{Tr} \int d^{3} x \bar{c}^{-2}\left(\partial^{\mu} B_{\mu}^{1}+\lambda^{1}\right) .
\end{aligned}
$$

\footnotetext{
${ }^{3}$ The dimension $(\mathrm{d})$ and the ghost number $(\Phi \Pi)$ of all fields are displayed in TABLE II.
} 
Moreover, since those four pieces (14) of the gauge-fixing shall break the on-shell nilpotency (10) of the BRS operator $s$, further modifications of the BRS transformations are necessary, however, in order to restore the BRS invariance an additional term has to be added to the gauge-fixing sector as well. The full modified gauge-fixing action,

$$
\Sigma_{\mathrm{gf}}=\Sigma_{\mathrm{gf}}^{1}+\Sigma_{\mathrm{gf}}^{2}+\Sigma_{\mathrm{gf}}^{3}+\Sigma_{\mathrm{gf}}^{4}+\Sigma_{\mathrm{mod}},
$$

with

$$
\Sigma_{\text {mod }}=\operatorname{Tr} \int d^{3} x \varepsilon^{\mu \nu \rho} \partial_{\mu} \bar{c}_{\nu}^{-1}\left[\partial_{\rho} \bar{c}^{-1}, B^{2}\right],
$$

reads

$$
\begin{aligned}
\Sigma_{\mathrm{gf}} & =\operatorname{Tr} \int d^{3} x\left\{b \partial^{\mu} A_{\mu}+\pi^{0} \partial^{\mu} B_{\mu}+\pi^{0 \nu}\left(\partial^{\mu} K_{\mu \nu}+\partial_{\nu} \rho^{0}\right)+\pi^{-1}\left(\partial^{\mu} B_{\mu}^{1}+\lambda^{1}\right)+\right. \\
& +\bar{c} \partial^{\mu} D_{\mu} c+\bar{c}^{-1} \partial^{\mu}\left(D_{\mu} B^{1}-\left[\phi, B_{\mu}^{1}\right]-\left[c, B_{\mu}\right]-\varepsilon_{\mu \nu \rho}\left[\partial^{\nu} \bar{c}^{-1 \rho}, B^{2}\right]\right)+ \\
& +\bar{c}^{-1 \nu}\left[\partial^{\mu}\left(D_{\mu} B_{\nu}^{1}-D_{\nu} B_{\mu}^{1}-\left[c, K_{\mu \nu}\right]-\varepsilon_{\mu \nu \rho}\left[\partial^{\rho} \bar{c}^{-1}, B^{2}\right]\right)-\partial_{\nu} \lambda^{1}\right]+ \\
& \left.+\bar{c}^{-2} \partial^{\mu}\left(D_{\mu} B^{2}+\left[c, B_{\mu}^{1}\right]\right)+\varepsilon^{\mu \nu \rho} \partial_{\mu} \bar{c}_{\nu}^{-1}\left[\partial_{\rho} \bar{c}^{-1}, B^{2}\right]\right\}
\end{aligned}
$$

where the enlarged BRS transformations associated to $B_{\mu}$ and $K_{\mu \nu}$ are given by

$$
\begin{aligned}
s B_{\mu} & =-D_{\mu} B^{1}+\left[\phi, B_{\mu}^{1}\right]+\left[c, B_{\mu}\right]+\varepsilon_{\mu \nu \rho}\left[\partial^{\nu} \bar{c}^{-1 \rho}, B^{2}\right], \\
s K_{\mu \nu} & =-\left(D_{\mu} B_{\nu}^{1}-D_{\nu} B_{\mu}^{1}\right)+\left[c, K_{\mu \nu}\right]+\varepsilon_{\mu \nu \rho}\left[\partial^{\rho} \bar{c}^{-1}, B^{2}\right] .
\end{aligned}
$$

Let us now introduce the action in which the nonlinear BRS transformations are coupled to the antifields (BRS invariant external fields), so as to control the renormalization of those transformations:

$$
\begin{aligned}
\Sigma_{\text {ext }} & =\operatorname{Tr} \int d^{3} x\left\{B_{\mu}^{*} s B^{\mu}+\frac{1}{2} K_{\mu \nu}^{*} s K^{\mu \nu}+A_{\mu}^{*} s A^{\mu}+\phi^{*} s \phi+c^{*} s c+B^{2 *} s B^{2}+B^{1 *} s B^{1}+B_{\mu}^{1 *} s B^{1 \mu}+\right. \\
& \left.+\frac{1}{2} \varepsilon^{\mu \nu \rho} B_{\mu}^{*}\left[K_{\nu \rho}^{*}, B^{2}\right]\right\} .
\end{aligned}
$$

The total classical action for the BFK-model, $\Gamma^{(0)}$ :

$$
\Gamma^{(0)}=\Sigma_{\mathrm{inv}}^{\mathrm{BFK}}+\Sigma_{\mathrm{gf}}+\Sigma_{\text {ext }},
$$

is invariant under the following BRS transformations

$$
\begin{gathered}
s B_{\mu}=-D_{\mu} B^{1}+\left[\phi, B_{\mu}^{1}\right]+\left[c, B_{\mu}\right]+\varepsilon_{\mu \nu \rho}\left[\partial^{\nu} \bar{c}^{-1 \rho}, B^{2}\right] \\
s K_{\mu \nu}=-\left(D_{\mu} B_{\nu}^{1}-D_{\nu} B_{\mu}^{1}\right)+\left[c, K_{\mu \nu}\right]+\varepsilon_{\mu \nu \rho}\left[\partial^{\rho} \bar{c}^{-1}, B^{2}\right], \\
s A_{\mu}=-D_{\mu} c, \quad s \phi=[c, \phi], \quad s c=c^{2}, \\
s B^{1}=\left[\phi, B^{2}\right]+\left[c, B^{1}\right], \quad s B_{\mu}^{1}=D_{\mu} B^{2}+\left[c, B_{\mu}^{1}\right], \quad s B^{2}=\left[c, B^{2}\right], \\
s \bar{c}=b, \quad s b=0 ; s \bar{c}^{-1}=\pi^{0}, \quad s \pi^{0}=0 ; \\
s \bar{c}^{-1 \mu}=\pi^{0 \mu}, \quad s \pi^{0 \mu}=0 ; s \bar{c}^{-2}=\pi^{-1}, \quad s \pi^{-1}=0, \\
s \rho^{0}=\lambda^{1}, \quad s \lambda^{1}=0 .
\end{gathered}
$$

The BRS invariance of the action (20) is expressed through the Slavnov-Taylor identity

$$
\begin{aligned}
\mathcal{S}\left(\Gamma^{(0)}\right) & =\operatorname{Tr} \int d^{3} x\left\{\frac{\delta \Gamma^{(0)}}{\delta B_{\mu}^{*}} \frac{\delta \Gamma^{(0)}}{\delta B^{\mu}}+\frac{1}{2} \frac{\delta \Gamma^{(0)}}{\delta K_{\mu \nu}^{*}} \frac{\delta \Gamma^{(0)}}{\delta K^{\mu \nu}}+\frac{\delta \Gamma^{(0)}}{\delta A_{\mu}^{*}} \frac{\delta \Gamma^{(0)}}{\delta A^{\mu}}+\frac{\delta \Gamma^{(0)}}{\delta \phi^{*}} \frac{\delta \Gamma^{(0)}}{\delta \phi}+\frac{\delta \Gamma^{(0)}}{\delta c^{*}} \frac{\delta \Gamma^{(0)}}{\delta c}+\frac{\delta \Gamma^{(0)}}{\delta B^{2 *}} \frac{\delta \Gamma^{(0)}}{\delta B^{2}}+\right. \\
& \left.+\frac{\delta \Gamma^{(0)}}{\delta B^{1 *}} \frac{\delta \Gamma^{(0)}}{\delta B^{1}}+\frac{\delta \Gamma^{(0)}}{\delta B_{\mu}^{1 *}} \frac{\delta \Gamma^{(0)}}{\delta B^{1 \mu}}+b \frac{\delta \Gamma^{(0)}}{\delta \bar{c}}+\pi^{0} \frac{\delta \Gamma^{(0)}}{\delta \bar{c}^{-1}}+\pi^{0 \mu} \frac{\delta \Gamma^{(0)}}{\delta \bar{c}^{-1 \mu}}+\pi^{-1} \frac{\delta \Gamma^{(0)}}{\delta \bar{c}^{-2}}+\lambda^{1} \frac{\delta \Gamma^{(0)}}{\delta \rho^{0}}\right\}=0,
\end{aligned}
$$

which translates, in a functional way, the invariance of the classical theory under the BRS symmetry. It is suitable to define, for later use, the linearized Slavnov-Taylor operator as below 


$$
\begin{aligned}
\mathcal{S}_{\Gamma^{(0)}} & =\operatorname{Tr} \int d^{3} x\left\{\frac{\delta \Gamma^{(0)}}{\delta B_{\mu}^{*}} \frac{\delta}{\delta B^{\mu}}+\frac{\delta \Gamma^{(0)}}{\delta B^{\mu}} \frac{\delta}{\delta B_{\mu}^{*}}+\frac{1}{2} \frac{\delta \Gamma^{(0)}}{\delta K_{\mu \nu}^{*}} \frac{\delta}{\delta K^{\mu \nu}}+\frac{1}{2} \frac{\delta \Gamma^{(0)}}{\delta K^{\mu \nu}} \frac{\delta}{\delta K_{\mu \nu}^{*}}+\frac{\delta \Gamma^{(0)}}{\delta A_{\mu}^{*}} \frac{\delta}{\delta A^{\mu}}+\frac{\delta \Gamma^{(0)}}{\delta A^{\mu}} \frac{\delta}{\delta A_{\mu}^{*}}+\right. \\
& +\frac{\delta \Gamma^{(0)}}{\delta \phi^{*}} \frac{\delta}{\delta \phi}+\frac{\delta \Gamma^{(0)}}{\delta \phi} \frac{\delta}{\delta \phi^{*}}+\frac{\delta \Gamma^{(0)}}{\delta c^{*}} \frac{\delta}{\delta c}+\frac{\delta \Gamma^{(0)}}{\delta c} \frac{\delta}{\delta c^{*}}+\frac{\delta \Gamma^{(0)}}{\delta B^{2 *}} \frac{\delta}{\delta B^{2}}+\frac{\delta \Gamma^{(0)}}{\delta B^{2}} \frac{\delta}{\delta B^{2 *}}+\frac{\delta \Gamma^{(0)}}{\delta B^{1 *}} \frac{\delta}{\delta B^{1}}+\frac{\delta \Gamma^{(0)}}{\delta B^{1}} \frac{\delta}{\delta B^{1 *}}+ \\
& \left.+\frac{\delta \Gamma^{(0)}}{\delta B_{\mu}^{1 *}} \frac{\delta}{\delta B^{1 \mu}}+\frac{\delta \Gamma^{(0)}}{\delta B^{1 \mu}} \frac{\delta}{\delta B_{\mu}^{1 *}}+b \frac{\delta}{\delta \bar{c}}+\pi^{0} \frac{\delta}{\delta \bar{c}^{-1}}+\pi^{0 \mu} \frac{\delta}{\delta \bar{c}^{-1 \mu}}+\pi^{-1} \frac{\delta}{\delta \bar{c}^{-2}}+\lambda^{1} \frac{\delta}{\delta \rho^{0}}\right\} .
\end{aligned}
$$

Since the BFK-model is a topological model of Schwarz type, in the Landau gauge, as we are assuming here, a symmetry called vector-supersymmetry [6, 13, 15] stems:

$$
\mathcal{W}_{\mu} \Gamma^{(0)}=\Delta_{\mu}^{\mathcal{W}_{\mu}}
$$

where its representation by means of a Ward operator is given by

$$
\begin{aligned}
\mathcal{W}_{\mu} & =\operatorname{Tr} \int d^{3} x\left\{-\varepsilon_{\mu \nu \rho}\left(\partial^{\nu} \bar{c}+A^{* \nu}\right) \frac{\delta}{\delta B_{\rho}}-\frac{1}{2} \varepsilon_{\mu \nu \rho} \phi^{*} \frac{\delta}{\delta K_{\nu \rho}}-\varepsilon_{\mu \nu \rho}\left(\partial^{\nu} \bar{c}^{-1}+B^{* \nu}\right) \frac{\delta}{\delta A_{\rho}}+\frac{1}{2} \varepsilon_{\mu \nu \rho}\left(\partial^{[\nu} \bar{c}^{-1 \rho]}+K^{* \nu \rho}\right) \frac{\delta}{\delta \phi}+\right. \\
& -A_{\mu} \frac{\delta}{\delta c}+B_{\mu}^{1} \frac{\delta}{\delta B^{2}}-B_{\mu} \frac{\delta}{\delta B^{1}}-K_{\mu \nu} \frac{\delta}{\delta B_{\nu}^{1}}-\bar{c}^{-2} \frac{\delta}{\delta \bar{c}^{-1 \mu}}+\partial_{\mu} \bar{c} \frac{\delta}{\delta b}+\partial_{\mu} \bar{c}^{-1} \frac{\delta}{\delta \pi^{0}}+\left(\partial_{\mu} \bar{c}_{\nu}^{-1}+\eta_{\mu \nu} \pi^{-1}\right) \frac{\delta}{\delta \pi_{\nu}^{0}}+ \\
& \left.+\partial_{\mu} \bar{c}^{-2} \frac{\delta}{\delta \pi^{-1}}+\partial_{\mu} \rho^{0} \frac{\delta}{\delta \lambda^{1}}-B^{1 *} \frac{\delta}{\delta B^{* \mu}}-\frac{1}{2} \eta_{\mu[\nu} B_{\rho]}^{1 *} \frac{\delta}{\delta K_{\nu \rho}^{*}}-c^{*} \frac{\delta}{\delta A^{* \mu}}-B^{2 *} \frac{\delta}{\delta B^{1 * \mu}}\right\}
\end{aligned}
$$

and the classical breaking (linear in the quantum fields), $\Delta_{\mu}^{\mathcal{W}_{\mu}}$, reads

$$
\begin{aligned}
\Delta_{\mu}^{\mathcal{W}_{\mu}} & =\operatorname{Tr} \int d^{3} x\left\{-B_{\nu}^{*} \partial_{\mu} B^{\nu}-\frac{1}{2} K_{\nu \rho}^{*} \partial_{\mu} K^{\nu \rho}-A_{\nu}^{*} \partial_{\mu} A^{\nu}-\phi^{*} \partial_{\mu} \phi+c^{*} \partial_{\mu} c-B^{2 *} \partial_{\mu} B^{2}+B^{1 *} \partial_{\mu} B^{1}+B_{\nu}^{1 *} \partial_{\mu} B^{1 \nu}+\right. \\
& \left.-\varepsilon_{\mu \nu \rho} B^{* \nu} \partial^{\rho} b+\varepsilon_{\mu \nu \rho} A^{* \nu} \partial^{\rho} \pi^{0}+\varepsilon_{\mu \nu \rho} \phi^{*} \partial^{\nu} \pi^{0 \rho}\right\} .
\end{aligned}
$$

By similar reasons to the existence of a vector-supersymmetry in the BFK-model, a scalar-supersymmetry can be found:

$$
\mathrm{E} \Gamma^{(0)}=\Delta^{\mathrm{E}}
$$

where its Ward operator reads

$$
\begin{aligned}
\mathrm{E} & =\operatorname{Tr} \int d^{3} x\left\{\frac{1}{2} \varepsilon_{\mu \nu \rho}\left(\partial^{\mu} \bar{c}+A^{* \mu}\right) \frac{\delta}{\delta K_{\nu \rho}}-\frac{1}{2} \varepsilon_{\mu \nu \rho}\left(\partial^{[\nu} \bar{c}^{-1 \rho]}+K^{* \nu \rho}\right) \frac{\delta}{\delta A_{\mu}}-\phi \frac{\delta}{\delta c}+B^{1} \frac{\delta}{\delta B^{2}}+B_{\mu} \frac{\delta}{\delta B_{\mu}^{1}}+\right. \\
& \left.-\bar{c}^{-2} \frac{\delta}{\delta \bar{c}^{-1}}+\pi^{-1} \frac{\delta}{\delta \pi^{0}}+B_{\mu}^{1 *} \frac{\delta}{\delta B_{\mu}^{*}}-c^{*} \frac{\delta}{\delta \phi^{*}}-B^{2 *} \frac{\delta}{\delta B^{1 *}}\right\},
\end{aligned}
$$

and its linear breaking in the quantum fields, $\Delta^{\mathrm{E}}$, is given by

$$
\Delta^{\mathrm{E}}=\operatorname{Tr} \int d^{3} x\left\{\frac{1}{2} \varepsilon_{\mu \nu \rho}\left(K^{* \mu \nu} \partial^{\rho} b-A^{* \mu} \partial^{[\nu} \pi^{0 \rho]}\right)\right\} .
$$

\section{E. Gauge conditions, ghost and antighost equations}

This Subsection is devoted to establish the gauge conditions, ghost and antighost equations, and two others quite important symmetries in the proof of the exact quantum scale invariance of the BFK-model.

The gauge conditions read

$$
\frac{\delta \Gamma^{(0)}}{\delta b}=\partial^{\mu} A_{\mu}, \quad \frac{\delta \Gamma^{(0)}}{\delta \pi^{0}}=\partial^{\mu} B_{\mu}, \quad \frac{\delta \Gamma^{(0)}}{\delta \pi^{0 \nu}}=\partial^{\mu} K_{\mu \nu}+\partial_{\nu} \rho^{0} \quad \text { and } \quad \frac{\delta \Gamma^{(0)}}{\delta \pi^{-1}}=\partial^{\mu} B_{\mu}^{1}+\lambda^{1},
$$

moreover, the conditions fulfilled by the BRS-doublet auxiliary fields, $\rho^{0}$ and $\lambda^{1}$, are given by 


$$
\frac{\delta \Gamma^{(0)}}{\delta \rho^{0}}=-\partial^{\mu} \pi_{\mu}^{0} \quad \text { and } \quad \frac{\delta \Gamma^{(0)}}{\delta \lambda^{1}}=-\partial^{\mu} \bar{c}_{\mu}^{-1}-\pi^{-1}
$$

The ghost equations:

$$
\begin{aligned}
& \mathcal{G}\left(\Gamma^{(0)}\right) \equiv \frac{\delta \Gamma^{(0)}}{\delta \bar{c}}+\partial^{\mu} \frac{\delta \Gamma^{(0)}}{\delta A^{* \mu}}=0, \quad \mathcal{G}^{1}\left(\Gamma^{(0)}\right) \equiv \frac{\delta \Gamma^{(0)}}{\delta \bar{c}^{-1}}+\partial^{\mu} \frac{\delta \Gamma^{(0)}}{\delta B^{* \mu}}=0, \\
& \mathcal{G}_{\mu}^{1}\left(\Gamma^{(0)}\right) \equiv \frac{\delta \Gamma^{(0)}}{\delta \bar{c}^{-1 \mu}}+\partial^{\nu} \frac{\delta \Gamma^{(0)}}{\delta K^{* \nu}}=-\partial_{\mu} \lambda^{1} \quad \text { and } \quad \mathcal{G}^{2}\left(\Gamma^{(0)}\right) \equiv \frac{\delta \Gamma^{(0)}}{\delta \bar{c}^{-2}}-\partial^{\mu} \frac{\delta \Gamma^{(0)}}{\delta B^{1 * \mu}}=0,
\end{aligned}
$$

mean that $\Gamma^{(0)}$ depends on the antighosts, $\bar{c}, \bar{c}^{-1}, \bar{c}_{\mu}^{-1}$ and $\bar{c}^{-2}$, and the antifields, $A_{\mu}^{*}, B_{\mu}^{*}, K_{\mu \nu}^{*}$ and $B_{\mu}^{1 *}$, through the combinations

$$
\widetilde{A}_{\mu}^{*}=A_{\mu}^{*}+\partial_{\mu} \bar{c}, \quad \widetilde{B}_{\mu}^{*}=B_{\mu}^{*}+\partial_{\mu} \bar{c}^{-1}, \quad \widetilde{K}_{\mu \nu}^{*}=K_{\mu \nu}^{*}+\partial_{[\mu} \bar{c}_{\nu]}^{-1} \quad \text { and } \widetilde{B}_{\mu}^{1 *}=B_{\mu}^{1 *}-\partial_{\mu} \bar{c}^{-2} .
$$

In the BFK-model there are two antighost equations, they are listed as below:

$$
\begin{aligned}
\overline{\mathcal{G}}\left(\Gamma^{(0)}\right) & \equiv \int d^{3} x\left\{\frac{\delta \Gamma^{(0)}}{\delta B^{1}}+\left[\bar{c}^{-1}, \frac{\delta \Gamma^{(0)}}{\delta b}\right]\right\}=\Delta^{\overline{\mathcal{G}}}, \text { where } \\
\Delta^{\overline{\mathcal{G}}} & \equiv \int d^{3} x\left\{\left[A^{\mu}, B_{\mu}^{*}\right]+\left[c, B^{1 *}\right]\right\}, \\
\mathbb{E}\left(\Gamma^{(0)}\right) & \equiv \int d^{3} x\left\{\frac{\delta \Gamma^{(0)}}{\delta B^{2}}-\left[\bar{c}^{-2}, \frac{\delta \Gamma^{(0)}}{\delta b}\right]\right\}=\Delta^{Æ}, \text { where } \\
\Delta^{\mathbb{E}} & \equiv \int d^{3} x\left\{\frac{1}{2} \varepsilon_{\mu \nu \rho}\left(\left[B^{* \mu},\left(\partial^{[\nu} \bar{c}^{-1 \rho]}+K^{* \nu \rho}\right)\right]+\left[K^{* \mu \nu}, \partial^{\rho} \bar{c}^{-1}\right]\right)+\left[B^{2 *}, c\right]+\left[B^{1 *}, \phi\right]+\left[B_{\mu}^{1 *}, A^{\mu}\right]\right\} .
\end{aligned}
$$

From those operators, $\overline{\mathcal{G}}$ and $Æ$, two others can be found by grading commutations with the Slavnov-Taylor operator (see the operatorial algebra in the next Subsection):

$$
\begin{aligned}
\overline{\mathcal{F}}\left(\Gamma^{(0)}\right) & \equiv \int d^{3} x\left\{\left[A_{\mu}, \frac{\delta \Gamma^{(0)}}{\delta B_{\mu}}\right]-\left[B_{\mu}^{*}, \frac{\delta \Gamma^{(0)}}{\delta A_{\mu}^{*}}\right]+\left[c, \frac{\delta \Gamma^{(0)}}{\delta B^{1}}\right]+\left[B^{1 *}, \frac{\delta \Gamma^{(0)}}{\delta c^{*}}\right]+\left[\bar{c}^{-1}, \frac{\delta \Gamma^{(0)}}{\delta \bar{c}}\right]+\right. \\
& \left.+\left[\pi^{0}, \frac{\delta \Gamma^{(0)}}{\delta b}\right]\right\}=0, \\
\varnothing\left(\Gamma^{(0)}\right) & \equiv \int d^{3} x\left\{-\frac{1}{2} \varepsilon_{\mu \nu \rho}\left(\left[\left(\partial^{[\mu} \bar{c}^{-1 \nu]}+K^{* \mu \nu}\right), \frac{\delta \Gamma^{(0)}}{\delta B_{\rho}}\right]+\left[\left(\partial^{\mu} \bar{c}^{-1}+B^{* \mu}\right), \frac{\delta \Gamma^{(0)}}{\delta K_{\nu \rho}}\right]\right)-\left[c, \frac{\delta \Gamma^{(0)}}{\delta B^{2}}\right]-\left[\phi, \frac{\delta \Gamma^{(0)}}{\delta B^{1}}\right]+\right. \\
& \left.-\left[A_{\mu}, \frac{\delta \Gamma^{(0)}}{\delta B_{\mu}^{1}}\right]-\left[\bar{c}^{-2}, \frac{\delta \Gamma^{(0)}}{\delta \bar{c}}\right]+\left[\pi^{-1}, \frac{\delta \Gamma^{(0)}}{\delta b}\right]+\left[B_{\mu}^{1 *}, \frac{\delta \Gamma^{(0)}}{\delta A_{\mu}^{*}}\right]+\left[B^{1 *}, \frac{\delta \Gamma^{(0)}}{\delta \phi^{*}}\right]-\left[B^{2 *}, \frac{\delta \Gamma^{(0)}}{\delta c^{*}}\right]\right\}=\Delta^{\varnothing}
\end{aligned}
$$

where $\Delta^{\varnothing} \equiv \int d^{3} x\left\{\frac{1}{2} \varepsilon_{\mu \nu \rho}\left(\left[\pi^{0}, \partial^{\mu} K^{* \nu \rho}\right]-\left[\pi^{0 \mu}, \partial^{[\nu} B^{* \rho]}\right]\right)\right\}$.

It should be pointed out that the breakings, $\Delta^{\overline{\mathcal{G}}}, \Delta^{Æ}$ and $\Delta^{\varnothing}$, being linear in the quantum fields are not subjected to renormalization.

\section{F. Operatorial algebra}

All operators introduced previously satisfy the following off-shell algebra for any functional $\mathcal{K}$ with even FaddeevPopov charge:

1. Slavnov-Taylor operator identities

$$
\begin{aligned}
& S_{\mathcal{K}} S(\mathcal{K})=0 \forall \mathcal{K}, \quad S_{\mathcal{K}} S_{\mathcal{K}}=0 \text { if } S(\mathcal{K})=0, \\
& \mathcal{W}_{\mu} S(\mathcal{K})+S_{\mathcal{K}}\left(\mathcal{W}_{\mu}(\mathcal{K})-\Delta_{\mu}^{\mathcal{W}_{\mu}}\right)=\mathcal{P}_{\mu}(\mathcal{K}), \quad \mathrm{\iota} S(\mathcal{K})+S_{\mathcal{K}}\left(\mathrm{E}(\mathcal{K})-\Delta^{\mathrm{E}}\right)=0, \\
& \frac{\delta S(\mathcal{K})}{\delta b}-S_{\mathcal{K}}\left(\frac{\delta \mathcal{K}}{\delta b}-\partial^{\mu} A_{\mu}\right)=\mathcal{G}(\mathcal{K}), \quad \frac{\delta S(\mathcal{K})}{\delta \pi^{0}}-S_{\mathcal{K}}\left(\frac{\delta \mathcal{K}}{\delta \pi^{0}}-\partial^{\mu} B_{\mu}\right)=\mathcal{G}^{1}(\mathcal{K}),
\end{aligned}
$$




$$
\begin{aligned}
& \frac{\delta S(\mathcal{K})}{\delta \pi^{0 \nu}}-S_{\mathcal{K}}\left(\frac{\delta \mathcal{K}}{\delta \pi^{0 \nu}}-\partial^{\mu} K_{\mu \nu}-\partial_{\nu} \rho^{0}\right)=\mathcal{G}_{\nu}^{1}(\mathcal{K})+\partial_{\nu} \lambda^{1}, \frac{\delta S(\mathcal{K})}{\delta \pi^{-1}}+S_{\mathcal{K}}\left(\frac{\delta \mathcal{K}}{\delta \pi^{-1}}-\partial^{\mu} B_{\mu}^{1}-\lambda^{1}\right)=\mathcal{G}^{2}(\mathcal{K}) \\
& \mathcal{G} S(\mathcal{K})+S_{\mathcal{K}} \mathcal{G}(\mathcal{K})=0, \mathcal{G}^{1} S(\mathcal{K})+S_{\mathcal{K}} \mathcal{G}^{1}(\mathcal{K})=0 \\
& \mathcal{G}_{\mu}^{1} S(\mathcal{K})+S_{\mathcal{K}}\left(\mathcal{G}_{\mu}^{1}(\mathcal{K})+\partial_{\mu} \lambda^{1}\right)=0, \mathcal{G}^{2} S(\mathcal{K})-S_{\mathcal{K}} \mathcal{G}^{2}(\mathcal{K})=0 \\
& \frac{\delta S(\mathcal{K})}{\delta \rho^{0}}-S_{\mathcal{K}}\left(\frac{\delta \mathcal{K}}{\delta \rho^{0}}+\partial^{\mu} \pi_{\mu}^{0}\right)=0, \frac{\delta S(\mathcal{K})}{\delta \lambda^{1}}+S_{\mathcal{K}}\left(\frac{\delta \mathcal{K}}{\delta \lambda^{1}}+\partial^{\mu} \bar{c}_{\mu}^{-1}+\pi^{-1}\right)=\frac{\delta \mathcal{K}}{\delta \rho^{0}}+\partial^{\mu} \pi_{\mu}^{0} \\
& \overline{\mathcal{G}} S(\mathcal{K})+S_{\mathcal{K}}\left(\overline{\mathcal{G}}(\mathcal{K})-\Delta^{\overline{\mathcal{G}}}\right)=\overline{\mathcal{F}}(\mathcal{K}), \quad \overline{\mathcal{F}} S(\mathcal{K})-S_{\mathcal{K}} \overline{\mathcal{F}}(\mathcal{K})=0, \\
& \mathbb{E} S(\mathcal{K})-S_{\mathcal{K}}\left(Æ(\mathcal{K})-\Delta^{Æ}\right)=\varnothing(\mathcal{K})-\Delta^{\varnothing}, \quad \varnothing S(\mathcal{K})+S_{\mathcal{K}}\left(\varnothing(\mathcal{K})-\Delta^{\varnothing}\right)=0
\end{aligned}
$$

\section{Other identities}

$$
\begin{aligned}
& \left\{\mathcal{W}_{\mu}, \mathcal{W}_{\nu}\right\}=0, \quad\left\{\mathcal{W}_{\mu}, \mathrm{E}\right\}=0, \quad\{\mathrm{E}, \mathrm{E}\}=0, \\
& \mathcal{W}_{\mu}\left(\overline{\mathcal{G}}(\mathcal{K})-\Delta^{\overline{\mathcal{G}}}\right)+\overline{\mathcal{G}}\left(\mathcal{W}_{\mu}(\mathcal{K})-\Delta_{\mu}^{\mathcal{W}_{\mu}}\right)=0, \quad \mathrm{E}\left(\overline{\mathcal{G}}(\mathcal{K})-\Delta^{\overline{\mathcal{G}}}\right)+\overline{\mathcal{G}}\left(\mathrm{E}(\mathcal{K})-\Delta^{\mathrm{E}}\right)=Æ(\mathcal{K})-\Delta^{Æ}, \\
& \mathcal{W}_{\mu} \overline{\mathcal{F}}(\mathcal{K})-\overline{\mathcal{F}}\left(\mathcal{W}_{\mu}(\mathcal{K})-\Delta_{\mu}^{\mathcal{W}_{\mu}}\right)=0, \quad \mathrm{€} \overline{\mathcal{F}}(\mathcal{K})-\overline{\mathcal{F}}\left(\mathrm{E}(\mathcal{K})-\Delta^{\mathrm{E}}\right)=\varnothing(\mathcal{K})-\Delta^{\varnothing}, \\
& \mathcal{W}_{\mu}\left(Æ(\mathcal{K})-\Delta^{Æ}\right)-\circledast\left(\mathcal{W}_{\mu}(\mathcal{K})-\Delta_{\mu}^{\mathcal{W}_{\mu}}\right)=0, \quad \mathrm{E}\left(Æ(\mathcal{K})-\Delta^{Æ}\right)-\circledast\left(\mathrm{E}(\mathcal{K})-\Delta^{\mathrm{E}}\right)=0, \\
& \mathcal{W}_{\mu}\left(\varnothing(\mathcal{K})-\Delta^{\varnothing}\right)+\varnothing\left(\mathcal{W}_{\mu}(\mathcal{K})-\Delta_{\mu}^{\mathcal{W}_{\mu}}\right)=0, \mathrm{\textrm {E }}\left(\varnothing(\mathcal{K})-\Delta^{\varnothing}\right)+\varnothing\left(\mathrm{E}(\mathcal{K})-\Delta^{\mathrm{E}}\right)=0, \\
& \overline{\mathcal{G}}^{a}\left(\overline{\mathcal{G}}^{b}(\mathcal{K})-\Delta^{\overline{\mathcal{G}} b}\right)+\overline{\mathcal{G}}^{b}\left(\overline{\mathcal{G}}^{a}(\mathcal{K})-\Delta^{\overline{\mathcal{G}} a}\right)=0, \quad \overline{\mathcal{F}}^{a} \overline{\mathcal{F}}^{b}(\mathcal{K})-\overline{\mathcal{F}}^{b} \overline{\mathcal{F}}^{a}(\mathcal{K})=0, \quad \overline{\mathcal{G}}^{a} \overline{\mathcal{F}}^{b}(\mathcal{K})-\overline{\mathcal{F}}^{a}\left(\overline{\mathcal{G}}^{b}(\mathcal{K})-\Delta^{\overline{\mathcal{G}} b}\right)=0, \\
& \overline{\mathcal{G}}^{a}\left(Æ^{b}(\mathcal{K})-\Delta^{Æ b}\right)-\circledast^{a}\left(\overline{\mathcal{G}}^{b}(\mathcal{K})-\Delta^{\overline{\mathcal{G}} b}\right)=0, \quad \overline{\mathcal{G}}^{a}\left(\varnothing^{b}(\mathcal{K})-\Delta^{\varnothing b}\right)+\emptyset^{a}\left(\overline{\mathcal{G}}^{b}(\mathcal{K})-\Delta^{\overline{\mathcal{G}} b}\right)=0, \\
& \overline{\mathcal{F}}^{a}\left(\mathbb{E}^{b}(\mathcal{K})-\Delta^{Æ b}\right)-\mathbb{E}^{a} \overline{\mathcal{F}}^{b}(\mathcal{K})=0, \overline{\mathcal{F}}^{a}\left(\varnothing^{b}(\mathcal{K})-\Delta^{\varnothing b}\right)-\varnothing^{a} \overline{\mathcal{F}}^{b}(\mathcal{K})=0,
\end{aligned}
$$

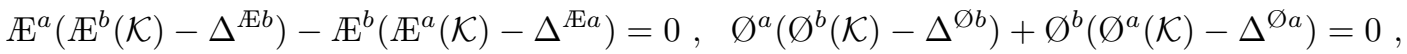

$$
\begin{aligned}
& \mathbb{E}^{a}\left(\varnothing^{b}(\mathcal{K})-\Delta^{\varnothing b}\right)-\varnothing^{a}\left(Æ^{b}(\mathcal{K})-\Delta^{Æ b}\right)=0, \\
& {\left[\mathcal{P}_{\mu}, \Theta\right]=0 \forall \Theta \in\left\{S_{\mathcal{K}}, \mathcal{W}_{\mu}, \mathrm{E}, \mathcal{G}, \mathcal{G}^{1}, \mathcal{G}_{\mu}^{1}, \mathcal{G}^{2}, \overline{\mathcal{G}}, \overline{\mathcal{F}}, \circledast, \varnothing, \mathcal{P}_{\mu}\right\},}
\end{aligned}
$$

where $\mathcal{P}_{\mu}$ is the Ward operator associated to translations:

$$
\mathcal{P}_{\mu}=\sum_{\varphi} \operatorname{Tr} \int d^{3} x \partial_{\mu} \varphi \frac{\delta}{\delta \varphi} .
$$

The first group of identities involving the Slavnov-Taylor operator given by (38) are those which yield the conditions (the well-known Wess-Zumino consistency condition is one of them) to be satisfied by the quantum breaking of the Slavnov-Taylor identity (22) allowed by the Quantum Action Principle [6].

\section{FINITENESS}

In this Section is summarized the results on the study of stability of the classical action under radiative corrections, the implementation of the Slavnov-Taylor identity at the quantum level and the conclusion on the finiteness of the BFK-model at all orders in perturbation theory.

\section{A. Stability}

In order to check whether the action in the tree-approximation is stable under radiative corrections or not, we perturb it by an arbitrary integrated local functional $\Sigma^{c}$, such that

$$
\widetilde{\Gamma}^{(0)}=\Gamma^{(0)}+\varepsilon \Sigma^{c},
$$




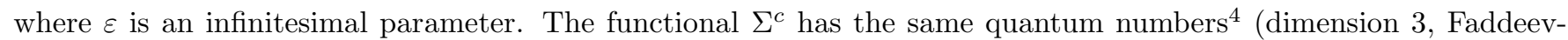
Popov charge $0, \ldots)$ as the classical action, $\Gamma^{(0)}$, and the deformed action, $\widetilde{\Gamma}^{(0)}$, must obey in the same way all the identities $\Gamma^{(0)}$ does, leading therefore, to the following homogeneous conditions to be fulfilled by the counterterm, $\Sigma^{c}$ :

$$
\begin{aligned}
& \mathcal{S}_{\Gamma^{(0)}} \Sigma^{c}=\mathcal{W}_{\mu} \Sigma^{c}=\mathrm{E} \Sigma^{c}=\frac{\delta \Sigma^{c}}{\delta b}=\frac{\delta \Sigma^{c}}{\delta \pi^{0}}=\frac{\delta \Sigma^{c}}{\delta \pi^{0 \mu}}=\frac{\delta \Sigma^{c}}{\delta \pi^{-1}}=\frac{\delta \Sigma^{c}}{\delta \rho^{0}}=\frac{\delta \Sigma^{c}}{\delta \lambda^{1}}=0, \\
& \mathcal{G} \Sigma^{c}=\mathcal{G}^{1} \Sigma^{c}=\mathcal{G}_{\mu}^{1} \Sigma^{c}=\mathcal{G}^{2} \Sigma^{c}=\overline{\mathcal{G}} \Sigma^{c}=Æ \Sigma^{c}=\overline{\mathcal{F}} \Sigma^{c}=\varnothing \Sigma^{c}=0 .
\end{aligned}
$$

In searching for the most general counterterm satisfying all the constraints listed above, it can be shown [17] that there is no integrated local polynomial in the fields which survives those requirements, then

$$
\Sigma^{c}=0,
$$

meaning that the usual ambiguities due to the renormalization procedure do not appear in the BFK-model. It remains now to prove the absence of anomalies in order to conclude about the perturbative finiteness of the model.

\section{B. Anomaly and finiteness}

At the quantum level the vertex functional, $\Gamma$, which coincides with the classical action (20), $\Gamma^{(0)}$, at order 0 in $\hbar$,

$$
\Gamma=\Gamma^{(0)}+\mathcal{O}(\hbar),
$$

has to satisfy the same constraints as the classical action does. However, according to the Quantum Action Principle [6] the Slavnov-Taylor identity (22) gets a quantum breaking

$$
\mathcal{S}(\Gamma)=\Delta \cdot \Gamma=\Delta+\mathcal{O}(\hbar \Delta),
$$

where $\Delta$ is an integrated local functional with ghost number 1 and dimension 3 . The absence of anomalies amounts to show that the Slavnov-Taylor identity can be implemented at the quantum level at the expenses of a BRS-trivial breaking $\Delta=\mathcal{S}_{\Gamma} \widehat{\Delta}^{(0)}$, called noninvariant counterterm.

The nilpotency identity, $S_{\Gamma} S(\Gamma)=0$, together with

$$
\mathcal{S}_{\Gamma}=\mathcal{S}_{\Gamma^{(0)}}+\mathcal{O}(\hbar)
$$

implies the Wess-Zumino consistency condition for the breaking $\Delta$ :

$$
\mathcal{S}_{\Gamma^{(0)}} \Delta=0,
$$

beyond that, through the algebra (38), $\Delta$ satisfies:

$$
\begin{aligned}
& \mathcal{W}_{\mu} \Delta=\mathrm{\textrm {L }} \Delta=\frac{\delta \Delta}{\delta b}=\frac{\delta \Delta}{\delta \pi^{0}}=\frac{\delta \Delta}{\delta \pi^{0 \mu}}=\frac{\delta \Delta}{\delta \pi^{-1}}=\frac{\delta \Delta}{\delta \rho^{0}}=\frac{\delta \Delta}{\delta \lambda^{1}}=0, \\
& \mathcal{G} \Delta=\mathcal{G}^{1} \Delta=\mathcal{G}_{\mu}^{1} \Delta=\mathcal{G}^{2} \Delta=\overline{\mathcal{G}} \Delta=\circledast \Delta=\overline{\mathcal{F}} \Delta=\varnothing \Delta=0 .
\end{aligned}
$$

The Wess-Zumino consistency condition (47) constitutes a cohomology problem in the sector of ghost number one. Its solution can always be written as a sum of a trivial cocycle $\mathcal{S}_{\Gamma^{(0)}} \widehat{\Delta}^{(0)}$, where $\widehat{\Delta}^{(0)}$ has ghost number 0 , and of nontrivial elements belonging to the cohomology of $\mathcal{S}_{\Gamma^{(0)}}(23)$ in the sector of ghost number one, $\mathcal{A}^{(1)}$ :

$$
\Delta=\mathcal{A}^{(1)}+\mathcal{S}_{\Gamma^{(0)}} \widehat{\Delta}^{(0)} .
$$

Although the constraints imposed to $\Delta$ in 477 and (48) show that $\mathcal{A}^{(1)}=0$ [17], it can be proved quite generally that in three-dimensions there is no anomaly, since the cohomology in the sector of ghost number 1 is empty up to possible terms in the Abelian ghosts [19]. However, through the arguments of [20] we conclude that the $U(1)$-ghosts do not contribute to the anomaly due to their freedom or soft coupling, then the Slavnov-Taylor identity is implemented at the quantum level.

In conclusion, the absence of counterterms in the study of stability, as presented in Subsection III A, together with the result of Subsection IIIB concerning the absence of anomaly lead to a proof on the finiteness of the BFK-model at all orders in perturbation theory.

\footnotetext{
${ }^{4}$ See TABLE for the dimension and the ghost number of all fields and anti-fields.
} 


\begin{tabular}{|c|c|c|c|c|c|c|c|c|c|c|c|c|c|}
\hline & $\overline{B_{\mu}}$ & $\overline{K_{\mu \nu}}$ & $\overline{A_{\mu}}$ & $\overline{\phi \phi}$ & $\bar{c}$ & $\bar{B}^{2}$ & $\overline{B^{1}}$ & $\bar{B}_{\mu}^{1}$ & $\overline{\bar{c}}$ & $\bar{c}$ & $\overline{\bar{c}^{-1}}$ & $\overline{\pi^{0}}$ & $\overline{\bar{c}_{\mu}^{-1}}$ \\
\hline$\Phi \Pi$ & 0 & 0 & 0 & 0 & 1 & 2 & 1 & 1 & -1 & 0 & -1 & 0 & -1 \\
\hline$\overline{c d}$ & $\overline{1}$ & 2 & 2 & $\bar{~} 1$ & $\begin{array}{ll}1 \\
\end{array}$ & 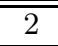 & 2 & 2 & 2 & 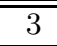 & $\overline{4}$ & 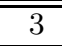 & $\overline{3}$ \\
\hline 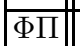 & 0 & -2 & -1 & 0 & 1 & -1 & -1 & -1 & -1 & -2 & -3 & -2 & -2 \\
\hline
\end{tabular}

TABLE I. Dimension $d$ and ghost number $\Phi \Pi$.

Acknowledgements: One of the authors (O.M.D.C.) thanks José A. Helayël-Neto, Susana I.Z. Caride, the Head of DCP-CBPF and Anibal O. Caride, the Head of CFC-CBPF, for financial support and the warm hospitality during all his visits at the Departamento de Teoria de Campos e Partículas (DCP) of the Centro Brasileiro de Pesquisas Físicas $(C B P F)$. He dedicates this work to his wife, Zilda Cristina, to his kids, Vittoria and Enzo, and his mother, Victoria. (J.M.G.) thanks Harald Ita for many useful discussions.

[1] D. Birmingham, M. Blau, M. Rakowski and G. Thompson, Phys.Rep. 209 (1991) 129; see also references therein.

[2] T. Fukuyama and K. Kanimura, Phys.Lett. B160 (1985) 259; K. Isler and C. Trugenberger, Phys.Rev.Lett. 63 (1989) 834.

[3] A.H. Chamseddine and D. Wyler, Phys.Lett. B228 (1989) 75 and Nucl.Phys. B340 (1990) 595.

[4] M. Blau and G. Thompson, Ann.Phys.(NY) 205 (1991) 130.

[5] A. Blasi and N. Maggiore, Class.Quant.Grav. 10 (1993) 37, hep-th/9207008.

[6] O. Piguet and S.P. Sorella, Algebraic Renormalization, Lecture Notes in Physics, m28, Springer-Verlag (Berlin-Heidelberg), 1995; see also references therein.

[7] R. Leitgeb, M. Schweda and H. Zerrouki, Nucl.Phys. B542 (1999) 425, hep-th/9904204.

[8] C. Lucchesi, O. Piguet and S.P. Sorella, Nucl.Phys. B395 (1993) 325, hep-th/9208047.

[9] M.I. Caicedo, R. Gianvittorio, A. Restuccia and J. Stephany, Phys.Lett. B354 (1995) 292, hep-th/9502137.

[10] M. Henneaux, Phys.Lett. B406 (1997) 66, hep-th/9704023.

[11] A. Accardi, A. Belli, M. Martellini and M. Zeni, Nucl.Phys. B505 (1997) 540, hep-th/9703152.

[12] O.M. Del Cima, D.H.T. Franco, J.A. Helayël-Neto and O. Piguet, J.High Energy Phys.(JHEP) 04 (1998) 010, hepth/9803247 and On the finiteness of BF-Yang-Mills theory in three dimensions, CBPF-NF-079/97, preprint (unpublished).

[13] F. Delduc, F. Gieres and S.P. Sorella, Phys.Lett. B225 (1989) 367; D. Birmingham and M. Rakowski, Mod.Phys.Lett. A4 (1989) 1753; D. Birmingham, M. Rakowski and G. Thompson, Nucl.Phys. B329 (1990) 83.

[14] O. Piguet, On the role of vector supersymmetry in topological field theories, talk given at the "XV Encontro Nacional de Física de Partículas e Campos", October 1994, Angra dos Reis, Brazil, hep-th/9502033.

[15] C.A.G. Sasaki, S.P. Sorella, O.S. Ventura and L.C.Q. Vilar, J.Math.Phys. 39 (1998) 848, hep-th/9706133.

[16] C.A.S. Almeida, R.R. Landim and D.M. Medeiros, Non-Chern-Simons topological mass generation in $(2+1)$ dimensions, hep-th/9906124.

[17] O.M. Del Cima, J.M. Grimstrup and M. Schweda, in preparation. Details can also be found in: M.Sc. Thesis (NBI), J.M. Grimstrup, Renormalization and finiteness of a generalized BF field theory, June 15, 1999, Copenhagen, Denmark.

[18] O.M. Del Cima, D.H.T. Franco, J.M. Grimstrup, O. Piguet and M. Schweda, work in progress.

[19] F. Brandt, N. Dragon and M. Kreuzer, Nucl.Phys. B340 (1990) 187; G. Barnich, F. Brandt and M. Henneaux, Nucl.Phys. B455 (1995) 357, hep-th/9505173.

[20] G. Bandelloni, C. Becchi, A. Blasi and R. Collina, Ann.Inst.Henri Poincaré 28 (1978) 225 and 255. 